\title{
PERIODONTAL DISEASE AS THE INITIAL ORAL MANIFESTATION OF LANGERHANS CELL HISTIOCYTOSIS = A CASE REPORT
}

A.R. Ahmad, s.M. Ismail, R.B. Zain, Z. Mohamad Zaini. Periodontal disease as the initial oral manifestation of Langerhans cell histiocytosis - a case report. Annal Dent Univ Malaya 2009; 16: 31-36.

\section{ABSTRACT}

Langerhans Cell Histiocytosis (LCH) refers to a group of lesions presenting with a spectrum of clinical features but sharing similar histology. These lesions are rare and treatment has been quite variable with current treatment protocol recommended being dependent on whether it is a unifocal or multi focal bone disease or a multi focal multisystem disease. However, the clinical presentations of $\mathrm{LCH}$ are variable and the decision to place into the appropriate clinical types may sometimes be masked by the non-discovery of all the lesions. In the oral maxillofacial area, the clinical features of these lesions may further pose a problem by nondescript manifestations as dental/periodontal/oral mucosal disorders. These oral findings may sometimes lead to inappropriate choice of treatment and delay in the diagnosis of all the lesions involved. This paper describes one such case where LCH manifest itself as a periodontal ... disease thus leading to delay in identifying all the sites involved and consequently a delay in id ntifying the appropriate clinical type

Key words: langerhans cell histiocytosis; oral disease; manifestation

\section{INTRODUCTION}

The term "Langerhans cell histiocytosis": (LCH) comprises three clinical overlapping syndromes which have morphologically similar lesions: Hand-SchullerChristian syndrome, Letterer-Siwe syndrome and localized eosinophilic granuloma. It is now regarded to represent as siqgle disease with a spectrum of clinical involvement ranged from a single localized lesion in a single organ to a fulminating systemic disease affecting multi focal and multisystem lesions (1-3). Recently, the differentiation between the 3 entities has been abandoned and the term "Langerhans cell histiocytosis" (LCH) is more frequently used. A recent review paper had discussed different classifications for the purpose of either treatment or prognostic assessment (4).

The cause of LCH is still poorly understood but all lesions consist of an abnormal proliferation of Langerhan's histiocytic cells which is different from the antigen presenting Langerhan cells with dendrites in normal epithelium. While both are derived from CD
Ca.se Report

A.R. Ahmadi, S.M. Ismaij2, R.B. Zainl,
Z. Mohamad Zaini I
LDepartment of Oral Pathology,
Oral Medicine and Periodontology,
Faculty of Dentistry,
University of Malaya,
Kual(l! Lumpur, t; talaysia
2 \{Jepartntent of Oral and Maxillofacial Surgery,
Faculty of DentistrY:
University of Malaya,
Kuala Lumpw; Malaysia
Corresponding author: Zuraiza Mohamad Zaini

34+ hematopoietic myel0id stem cells In the bone mar ow, Langerhans histiocytic cells are immature qenctfitic cells $\sim$ here their ability to present antigen is either Ipst 91' not observed (5-6). Most of LCH cases were commonly seen in the pediatric patients with a peak incidence before the third year of age (I). In adults, more than $50 \%$ of ca'ses were found in patients above the second decade (2-3). The clinical course and prognosis of the disease depend on the age of initial manifestation as well as the number and sites of the involved organs (1-3). Prominent locations are the flat ones, such as the ribs, the spine and the cranial bone. In this case report, we present a case where the clinical features first appeared in the oral cavity, thus mimicking a periodontal disorder.

Case report

A 20-year old Indian male presented to a dental practitioner because several of his lower teeth were steadily becoming very loose and tender for the last few months. He was diagnosed with chronic periodontal disease by his general dental practitioner. Both the left and right s'econd mandibular premolars were extracted on the same day under local anesthesia ithout any pre-operative ;adiographic assessment. The socket failed to heal after one week. He returned to the same dentist and was given a week's course of antibiotic without improvement. Different types of antibiotics were later prescribed,.., with no significant improvement. Eventually the patient was referred to the F culty of Dentistry, University of Malaya for management of the non-healing extraction sockets of the left: and right second premolars. The patient was generally healthy and his previous medical history was unremarkable. The patient was a smoker and occasional alcohol drinker. Hematological investigations (full 


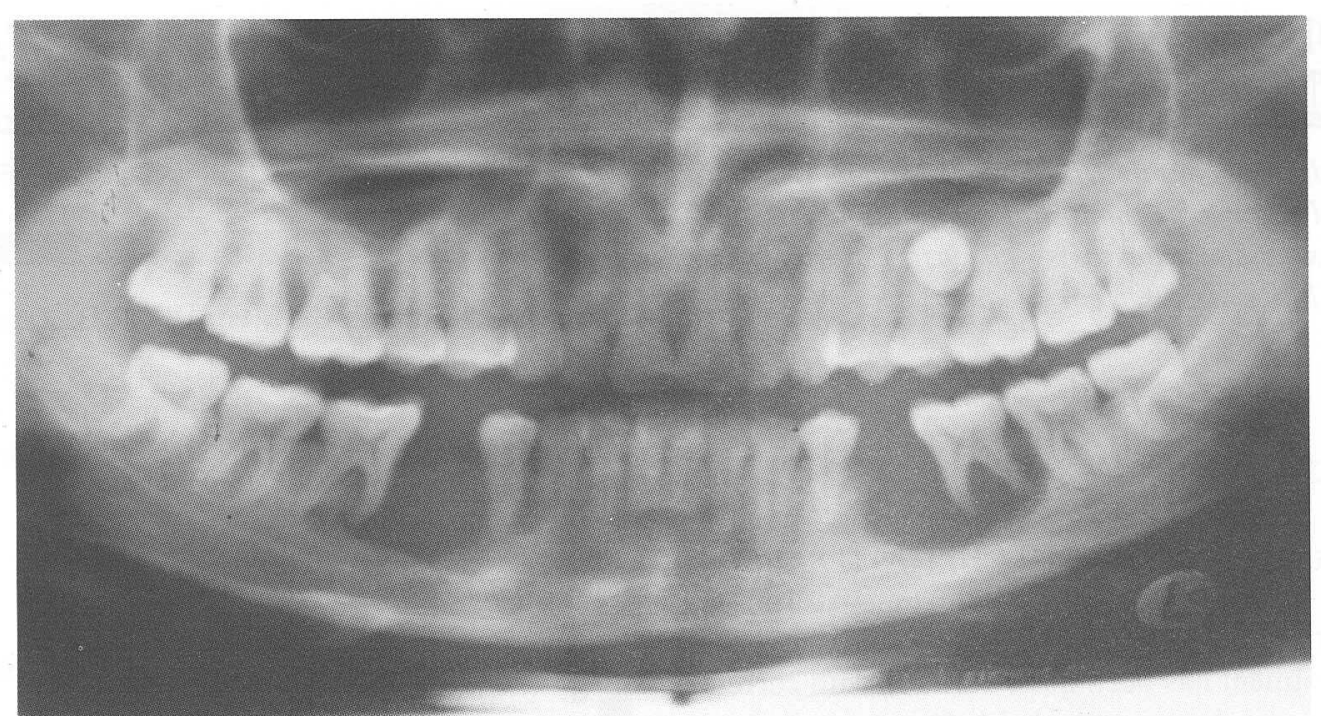

Figure 1. An OPO view showing bilateral radiolucencies indicative of alveolar bone loss involving the right and left: mandible and floating of the 36 tooth

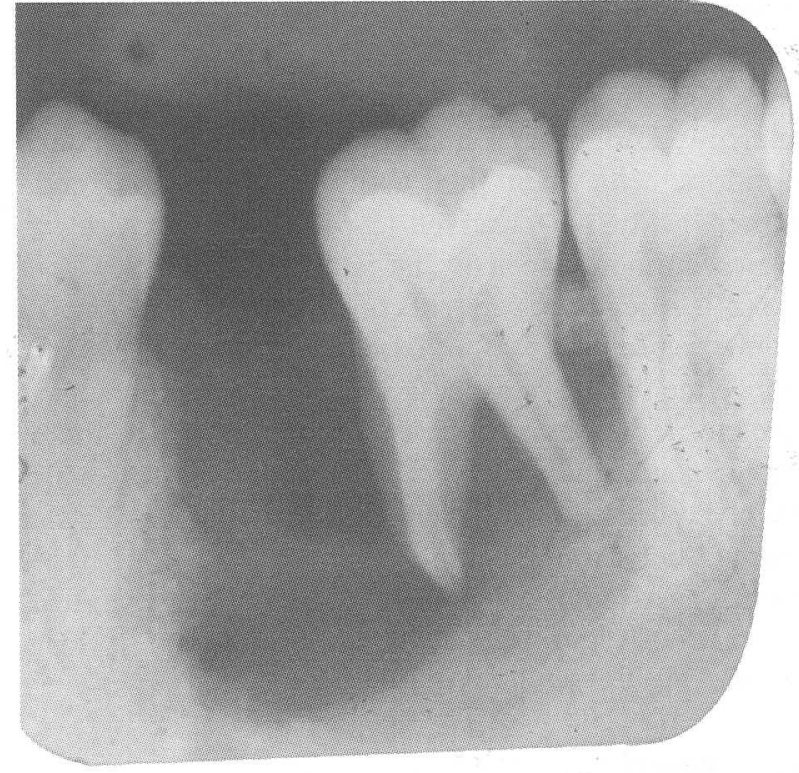

Figure 2..A periapical view owed gross alveolar b0 ne lost involving the 36 root region adjacenUo the non-healing

35 extraction socket. A 'scQoped out' bone lesion was seen at the distal root of $3-6$.

blood count $[\mathrm{FBC}]$, liver function test $[\mathrm{LFT}]$ and $\sim$ nal function test [RFT]) showed all factors wefe within normal limits.

\section{Clinical Presentation}

A clinical examination of the oral cavity showed the sites of previous extraction were inflamed with patent cavities filled with food debris. Both the right and left first lower molars were extremely mobile. Several other teeth in the right and left mandible showed abnormal mobility and abnormal gingival pocket depths of more than five millimeters. Marked gingival pocket depth was also detected in between the upper right first and second molars. A panoramic radiograph revealed a large and well demarcated radiolucent lesions in the right and left mandible (Figure I) with the left first lower molar appeared 'floating' in the lesion (Figure 2). A differential diagnosis of eosinophilic granulom , aggressive periodontal disease, and malignancy was made. A surgical curettage with removal of the right and left first lower molars was performed.

\section{Histopathology}

Histopathological examination of the excisional biopsy specimen showed sheets of Langerhans cells with a mixed infiltrate of macrophages, eosinophils and lymphocytes (Figure 3). Immunohistochemically, the Langerhan cells were strongly positive for S 100 p"rotein and CDla (Figures 4 and 5).

\section{Diagnosis}

A diagnosis of eosinophilic granuloma was established based on the histological and radiographic findings of the lesions in the mandible.

\section{Treatment}

Since the patient was generally healthy, the case was treated as a localized LCH of the mandible where surgical curettage was done. Close and regular followup for a year were carried out showing no progression of the lesions.

At the end of the I year follow-up, the upper right first and second molars were mobile and the patient was further treated for a concomitant periodontal disease where generalized scaling and root planning of the upper right first and second molars were performed. However, a poor response to the periodontal treatment was noted in the right upper first and second molars and, a biopsy of the area was performed. Similar histology as the earlier excisional biopsy showing features of $\mathrm{LCH}$ was seen. A full and thorough body 


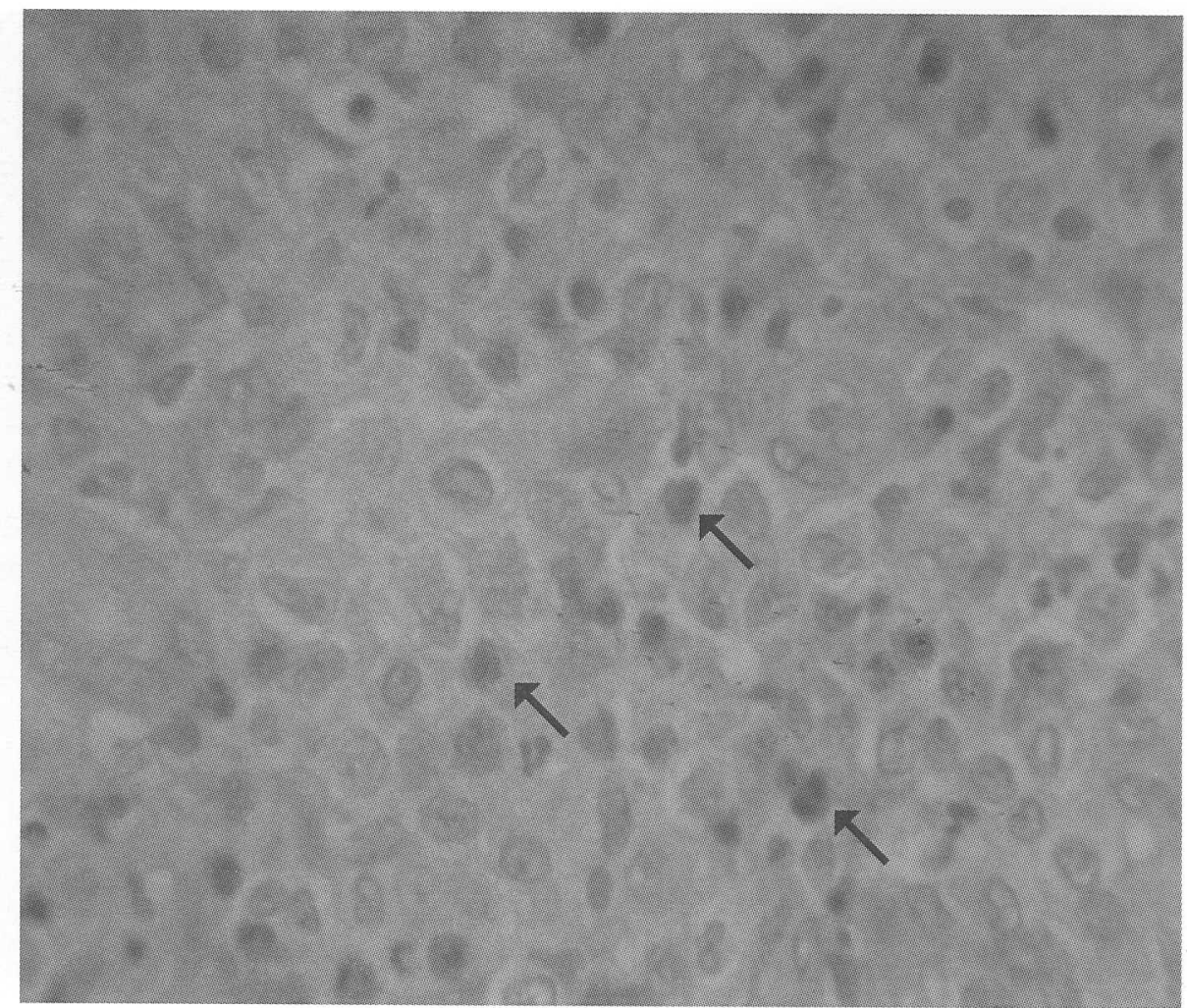

Figure 3. H\&E: Section shows sheet of histiocyte-like-cells with scattered eosinophils (arrows) (40X original magnification)_

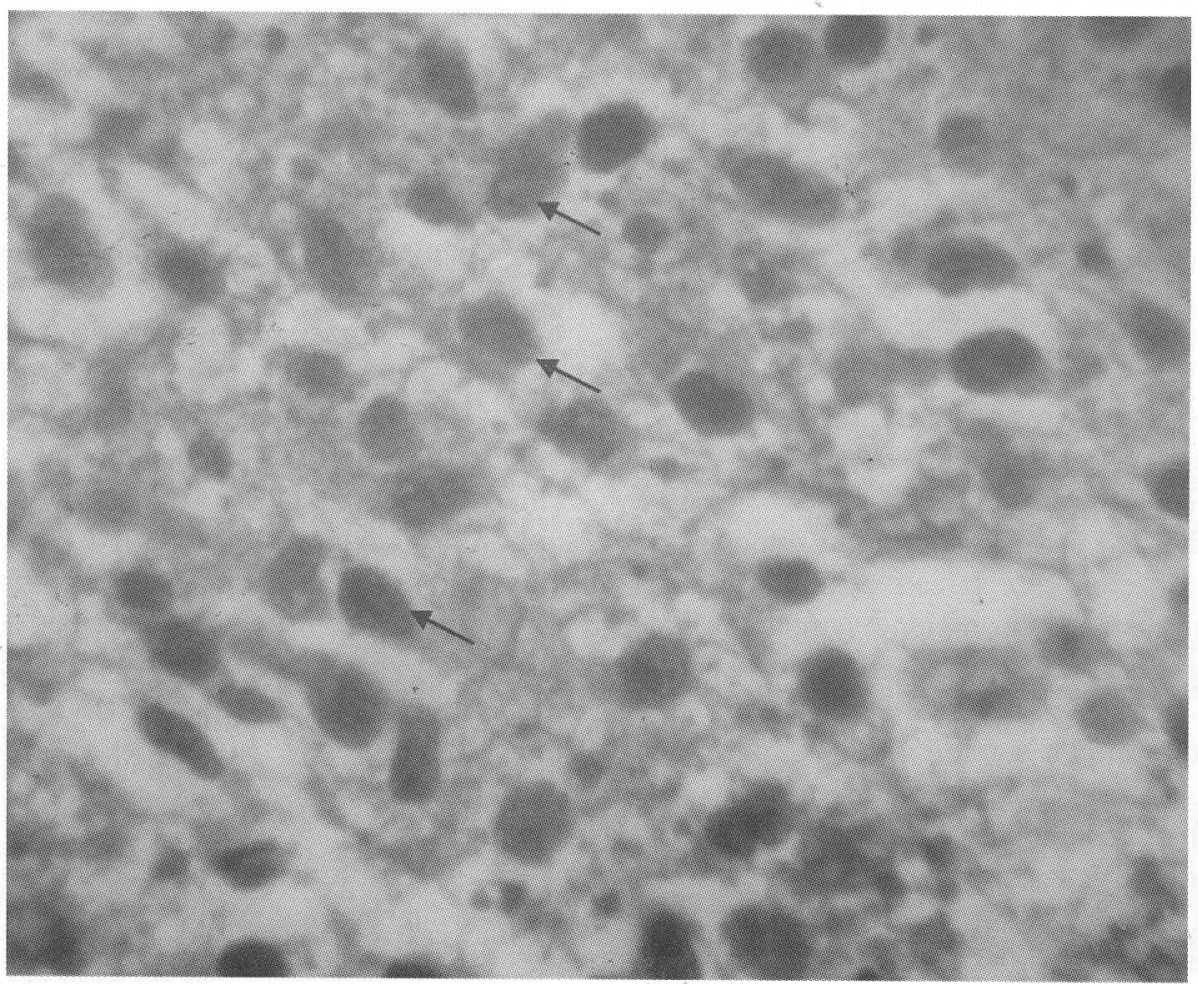

Figure 4. Positive S-IOO immunostaining in nucleus and cytoplasm of Langerhan cells (arrows) (40X original magnification). 


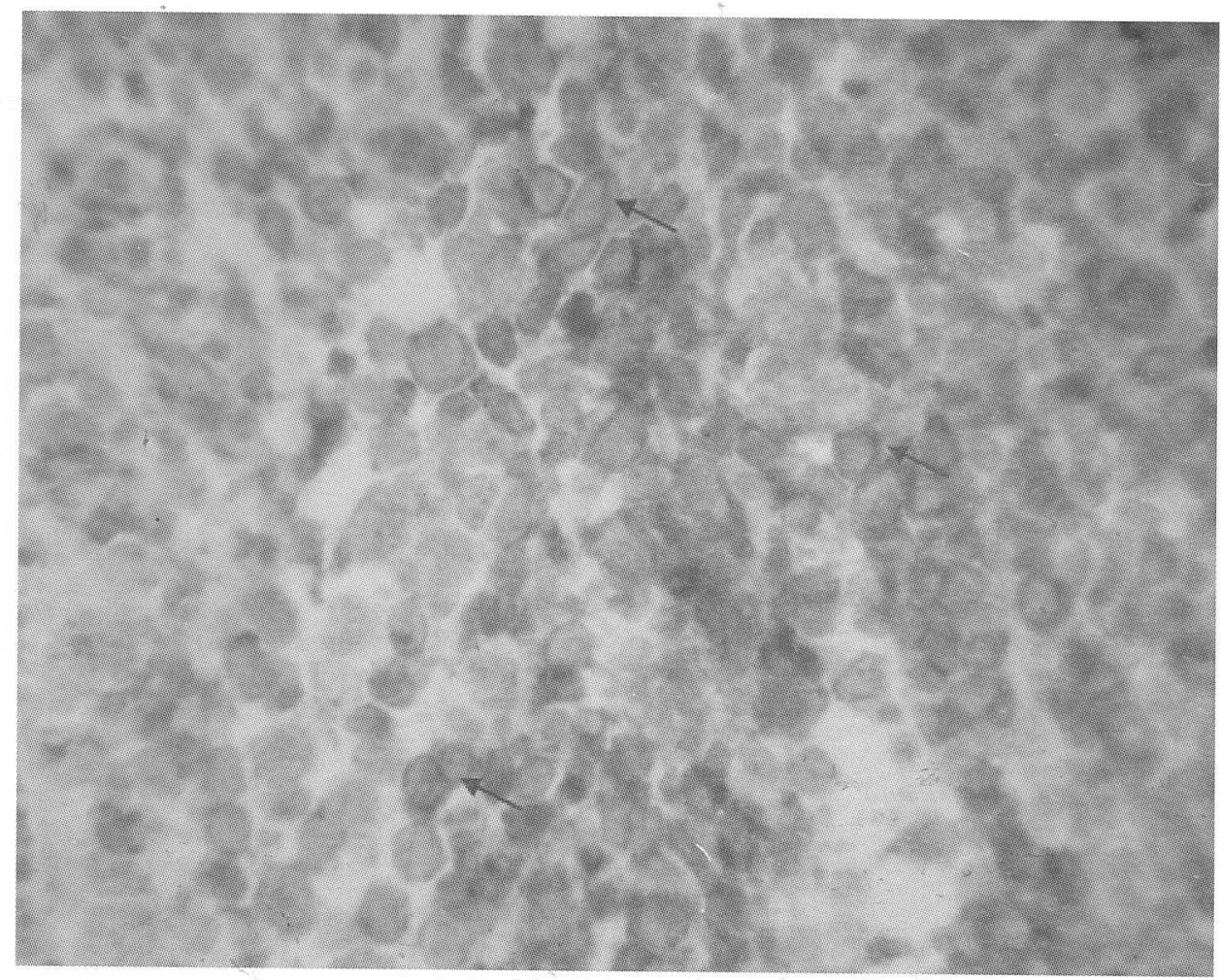

Figure 5. Positive CD Ia immunostaining in cytoplasm of Langerhan cells (arrows) (40X original magnification).

examination was performed and a chest radiograph revealed a large lesion in the lung. Tbe somputerized t0mography (CT) scan was perf9fmed and confirmed the presence of the lung lesion. The latter finpings lead to a change in the clinical types from multi focal bone le sions to a multifocal multisystem type of $\mathrm{LCH}$ and thus the patient was further managed by an oncologist with chemotherapy.

\section{DISCUSSION}

Langerhan's cell histiocytosis (LCH), form rly known as histiocytosis $\mathrm{X}$, is characte\{"ized by abnormal proliferation - of Langerha.ns cells. Jt can involve virtually any site and organ of the body, either as an isolated lesion or as a wide spread systemic disease. Involvements of the liver, spleen, lung, lymphoidlissue and skin have been reported (1-3). B.91J can be involved as a part of generalized disease or as a separate entity, known as localized Langerhans histiocytosis. Localized Langerhans cell histiocytosis, formerly known as eosinophilic granuloma account for about $40-50 \%$ of all reported cases of Langerhans cell histiocytosis in the adult (5). A single bone or multiple bones can be affected. The skull, pelvic and ribs are the most frequently affected in children. In adults, vertebrae and long bones are most frequently affected (1-4).The highest incidence of the disease is in the first three decades of life, with males being affected twice as frequently as female (5).
Involvement of the jaws either as a localized $\mathrm{LCH}$ or with visceral involvement has been reported (7). The age of occurrence is usually after 20 years of age (8I I). Involvement of the alveolar bone alone was reported in more than $50 \%$ of all $\mathrm{LCH}$ cases involving the mandible or maxilla (11). Involvement of the alveolar bone alone without an intra-osseous lesion at other sites may pose a diagnostic problem as the lesion may mimic routine periodontitis. This was true in early or small lesions of LCH where the pattern of alveolar bone lost cannot be differentiated clinically or radiographically from periodontitis. In our current case report, the involvement of the jaws first manifested as mobile lower left and right premolars thus mimicking clinical periodontal disease. A study by Artzi and Raviv (12) reported that 28 cases of LCH cases exhibited signs and symptoms suggestive of periodontal disease. Cranin and Rockman (13) described 3 cases of LCH presenting with loose molars, periodontal disease and precocious eruption of the primary dentition with gingival bleeding. Alajbeg et all (14) described a case of oral LCH that was not recognized until systemic progression of the di;; ease.

Radiographically, bony lesions often appear as sharply punched out radiolucencies without a sclerotic or corticated margin. Occasionally, an ill-defined radiolucency was seen. Bone involvement in the mandible usually occurs in the posterior areas, and a characteristic "scooped out" appearance may be evident when superficial alveolar bone is destroyed. The resulting bone destruction and loosening of the teeth 
clinically may resemble severe periodontitis. Extensive alveolar involvement causes the teeth to appear as if they are 'floating in the space' (11). When the orthopantomogram of our case was taken, there were the characteristic 'scooped out' lesions around the left and right molars with these teeth appearing as 'floating in space'. In less typical raCliographic appearances, a number of differential diagnosis namely chronic osteomyelitis, "aggressive periodontal disease, odontogenic cyst or malignancy may be considered (8-9).

In gener 1, the diagnosis of LCH is based on certain morphological, immunohistochemical, and/or ultra structural features. The disorder is characterized by the abnormal proliferation of large cell with an abundance of cytoplasm, indistinct cell borders with oval to reniform nuclei. These cells are most often arranged in sheets and may be intermixed with substantial numbers of eosinophils (8).

We based our histological diagnosis on the morphological features and the positivity of the Langerhans cell histiocytes when immunohistochemically stained with S100 and CD 1a. This is in keeping with the criteria of the Histiocyte Society, which requires that the histopathological diagnosis of $\mathrm{LCH}$ is established with the conventional histology and at least 2 positive stainings for ATP-ase, S-IOO, alphaD-mannosidase (CD 1a) or peanut lectin (PNA). The presence of intracytoplasmic Birbeck granules on ultrastructural analysis may further support the diagnosis. However, several studies reported that not all cases of CD 1a-positive Langerhans cells showed presence of this structure (15-16). The use of S-100 alone may be insufficient to establish the diagnosis of $\mathrm{LCH}$ since it is also expressed by the cells of peripheral: nervous system and melanocytes. CDla on the other hand, is also expressed by cell surfaces of cortical thymocytes and interdigitating dendritic cells (15). Taken together; both S-100 and CD 1a immunostaining must be carried out to establish diagnosis ofLCH (15).

Surgery, chemotherapy, and radiotherapy have been used alone or in combination to treat $\mathrm{LCH}$ of the jaws. Most of accessible bone lesions, such as those in the maxilla and mandible, are found to be adequately treated by curettage alone $(9,17)$. Low dose radiation may be employed for less acc ssible bone lesions and in cases where curettage alone failed to completely control the lesions. However in younger patient, there is a need to consider the potential of induction of malignant changes secondary to this treatment (18). In several cases, adjuvant chemotherapy was found to be effective in recurrent lesions after surgical curettage (9). Intralesional injection with corticosteroid has been reported to be effective in some patients with multiple bone lesions in the jaws (19).

Multiple organ involvement or multiple bone lesions usually requires multi-modalities of treatment and should be managed by an oncologist (9). The prognosis for bone lesions in the absence of significant visceral involvement is generally good; however, progression or dissemination of the disease may occur (9, 15).

In this current reported case of LCH, an enucleation was first performed which is in keeping with the findings of a multifocal bone disease. However, upon the discovery of the lesions in the lung, the management should be changed to the clinical type of multifocal multisystem disease. The combination 'of surgery and chemotherapy is partly in-keeping with the proposed management strategies for such a clinical ondition $(4,9)$. The recommended treatment for this clinical subtype is a combination of surgery, chemotherapy and radiotherapy. The 'pulmonary ciisease only' represents the third clinical subtype where the recommended treatment is prednisone and/or ch motherapy and/or surgery.

According to the review by Hicks and Flaitz (4), the combination of surgery, chemotherapy and radiation for th treatment of multifocal multisystem type has led to $74 \%$ of disease-free survival, $11 \%$ alive with disease and $15 \%$ died of disease. However, relapse occurs in about $95 \%$ of cases.

\section{CONCLUSION}

$\mathrm{LCH}$ is an uncommon disease and this cjlse report is !ntended to show that LCH at an early sfage can present as chronic periodontal disease. Therefore, radiographic interpretation is extremely important before any final diagnosis and treatment of a periodontal disease is made in cases of unexplained mobility of tooth/teeth.

\section{REFERENCES}

1. Cochrane LC, Prince M, Clarke K. Lange,rhans in paediatric population:presentation and treatment of head and neck manifestation. J Otolaryngol 2003; 32: $33-7$.

2. Malpas JS, Norton AI. Langerhans cell histiocytosis in the adult. Med Pediatr Onco11996; 27: $540-6$.

3. Baumgartner I, von Hochstetter A, Baumert B, Luetolf U, Follath F. Langerhans cell histiocytosis in adults. Med Pediatr Onco11997; 28: 9-14.

4. Hicks J, Flaitz, CM. Langerhans cell histiocytosis: Current insights in a molecular age with emphasis on clinical oral and maxillofacial pathology practice. Oral Surg Oral Med Oral Pathol Oral Radiol Endod 2005; 100: 42-66.

5. Chu T, Jaffe R. The normal Langerhans cell and the LCH cells. Br J Cancer 1994; 23: 4-10. 
6. Lombardi T, Hauser C, Budtz-Jorgensen E. Langerhans cell: structure, function and role in Qra] pathological condition. J Oral Pathol Med 1993; 22: ] $93-202$.

7. Yasko AW, Fa ning $\mathrm{Cv}$, Aya]a AG. Percutaneous techniques for the diagnosis and treatment of Jocalized Langerhans' cell histiocytosis eosinophIlic granuloma of bone. J Bone Joint Surg 1998; 80: 219-28.

8. Hartman KS. Histiocytosis X: A review of 114 cases with ora] involvement. J Oral Surg 1980; 49(1): 38-54.

9. Eckardt A, Schultze A. Maxillofacia] manifestation of Langerhans cell histiocytosis: a clinical and therapeutic analysis of 10 patients. Oral Oneol 2003; 39: 687-94.

10. Arico M. Langerhans cell histiocytosis in adults: more questions than answer? E, ur J Cancer 2004; 40: ]467-73.

11. Dagenais M, Pharoah MJ, Siroski PA. The radiographic characteristics of histiocytosis X. A study of 29 cases that involve the jaws. Oral Surg Oral Med Orell Patho11992; 74: 230-6.

If. Artzj Z, Raviv M. Periodontal manifestations of adult onset of histiocytosis X. J Pe/'iodontol 1989; 60: 57.

13. Cranin AN, Rockman R. Oral symptom in LCH. J Am Dent Assoe 1981; 103: 412-6 .
14. Alajbeg I, Boras 'Iv, , Feminic R, Cekic-Arambasin A, Anicic M, Kelecic J, Alajbeg IZ. Unrecognized oraL manifestation of Langerhans cell histiocytosis which progressed to systemic disease. Oral Oneol 2006; 42: 10-3.

15. Krenacs L, Tiszlavicz L, Krenacs T. Immunohistochemical detection of CD] a antigen in formalin-fixed and parafftn-embedded tissue sections with monoclonal antibody. J Pathol1993; 171: $99-104$

16. Takahashi K, Harada M, Kimoto M, Kondo F. Diagnostic confirmation of Jangerhans cell histiocytosis of the jaws with CDla immunostaining: A case report. J Oral Maxillófae 2003; 61: 118-22.

17. Bartnick A, Friedrich RE, Roeser K, Schmelzle R. Ora] Langerhans cell histiocytosis ..J CranioMaxillofae Surg 2002; 30:--91-6.

18. Howarth DM., Gilchrist GS, Mullan BP. Langerhans cell histiocytosis. D;agnosis, natural history, management,.. and outcome. Cancer 1999; 85: $2278-90$.

19. Putters TF, de Visscher A, van Veen A, Spijkervet FKL. Intralesional infiltration of corticosteroids in the treatment of Jocalised langerhans cell histiocytosis of the mandible: Report of known ases and three new cases. Int J Oral Maxillofae Surg 2005; 34: 571-5. 